\title{
Viscoelastic coagulation test for liver transplantation
}

\section{Sun Young Park}

Department of Anesthesiology and Pain Medicine, Soonchunhyang University Seoul Hospital, Seoul, Korea
Received March 2, 2020

Accepted March 5, 2020

\author{
Corresponding author \\ Sun Young Park, M.D., Ph.D. \\ Department of Anesthesiology and \\ Pain Medicine, Soonchunhyang \\ University Seoul Hospital, 59 \\ Daesagwan-ro, Yongsan-gu, Seoul \\ 04401, Korea \\ Tel: 82-2-709-9291 \\ Fax: 82-2-790-0394 \\ E-mail: sunnypark97@schmc.ac.kr
}

Coagulation and transfusion management in patients undergoing liver transplantation is challenging. Proper perioperative monitoring of hemostasis is essential to predict the risk of bleeding during surgery, to detect potential causes of hemorrhage in time, and to guide hemostatic therapy. The value of conventional coagulation test is questionable in the acute perioperative setting due to their long turnaround time and the inability to adequately reflect the complex changes in hemostasis in patients with liver disease. Viscoelastic coagulation tests provide simultaneous measurement of multiple aspects of whole-blood coagulation including plasmatic coagulation and fibrinolytic factors and inhibitors that reflect most aspects of hemostasis. Coagulation initiation, mechanical clot stability, and fibrinolysis can be estimated immediately using point-of-care techniques. Therefore, viscoelastic coagulation tests including ROTEM \& TEG would be useful to guide patient blood management strategy during liver transplantation.

Keywords: Blood coagulation disorders; Blood coagulation tests; Liver transplantation; Thromboelastography.

\section{INTRODUCTION}

As the liver plays an important role in the blood coagulation system, hepatic dysfunction could lead to coagulopathy. Even during hepatic failure, the coagulation system can maintain a balance via various mechanisms, but it is not stable. Most patients undergoing liver transplantation experience severe changes in blood coagulation status during surgery that results in bleeding tendency. Because excessive bleeding and blood transfusion during liver transplantations are risk factors for patients' poor outcomes [1], it is important to rapidly perceive and manage the changes of the blood coagulation status to prevent excessive bleeding and reduce blood transfusion during surgery. Conventional coagulation test (CCT) to diagnose coagulation disorders includes tests for bleeding time (BT), prothrombin time (PT), activated partial prothrombin time
(aPTT), thrombin time (TT), platelet count, and fibrinogen. However, studies have reported that it is difficult to predict the possibility of bleeding or blood transfusion with CCT [2-4] and that CCT is insufficient to distinguish whether a coagulopathy has occurred $[5,6]$. Furthermore, CCT has the disadvantage of being time-consuming, and hence, it cannot be applied in situations that require rapid judgment and treatment. Recently, viscoelastic coagulation test has been garnering attention as a method that can overcome these limitations [7]. This test was developed in 1948 and approved for use in enabling targeted treatment by distinguishing deficient coagulation factors in patients with coagulation factor deficiencies. Since the 1960s, cases of perioperative applications have been reported. Viscoelastic coagulation test is a method to determine coagulation in real-time by obtaining the thromboelastrography while the blood coagulation is in process. It is reported that this test

This is an Open Access article distributed under the terms of the Creative Commons Attribution Non-Commercial License (http://creativecommons.org/licenses/by-nc/4.0) which permits unrestricted non-commercial use, distribution, and reproduction in any medium, provided the original work is properly cited.

Copyright (C) the Korean Society of Anesthesiologists, 2020 
can provide a more comprehensive and accurate information about coagulation function than conventional tests [8]. Other than the platelet count, most CCTs are plasma-based tests and can only reflect the initial stages of the coagulation. In contrast, viscoelastic coagulation tests are plasma and cell-based tests using the whole blood and can provide information from coagulation initiation to fibrinolysis, the strength and stability of the clot. Therefore, it provides a real time accurate biological representation of coagulation [6] and can be used as a point-of-care testing. The viscoelastic coagulation test is often applied to patients undergoing surgery for trauma in which bleeding can be problematic, and various studies on its utility has been conducted. And there are many studies have been conducted on its use during liver transplantation recently. Therefore, this review article aims to examine the usefulness of the viscoelastic coagulation test and its application in liver transplantation.

\section{VISCOELASTIC COAGULATION TEST}

Viscoelastic coagulation test is a method that measures, digitalizes, and graphically plots the viscoelasticity generated between the fibrin strands and the platelets in the process of blood coagulation. There are currently two types of equipment that can be used for viscoelastic coagulation test: classical thrombelastography (TEG, Haemonetics, USA) and rotational thromboelastometry (ROTEM, Tem International GmbH, Germany). The parameter that can be obtained first through these tests is the clotting time (clotting time or CT in ROTEM; reaction time or $\mathrm{R}$ in TEG) which is the time it takes until the graph amplitude reaches $2 \mathrm{~mm}$. This can be considered an index of the coagulation factor function. Delay in this parameter can signal coagulation factor deficiency or dysfunction. The next parameter is the amount of time it takes for the amplitude to increase from $2 \mathrm{~mm}$ to $20 \mathrm{~mm}$ which is referred to as the clot formation time (clot formation time or CFT in ROTEM; $\mathrm{k}$ in TEG), and this reflects fibrin formation. Platelet response is considered to start at this time, and the slope of the graph until this point is represented as the $a$ angle. Then, the amplitude gradually increases and reaches maximum. This maximum amplitude (maximum clot firmness or MCF in ROTEM; maximum amplitude or MA in TEG) is an index that reflects the strength of the clot. To quickly assess the strength of the clot, estimates can be made from the amplitudes measured at the 5, 10, and $20 \mathrm{~min}$ after the clotting time. The final parameter represents the fibrinolysis. The amplitude $30 \mathrm{~min}$ or $60 \mathrm{~min}$ after clotting time is calculated as a percentage of the maximum amplitude (LI30, LI60 in ROTEM; LY30, LY60 in TEG) to represent the stability of the clot (Fig. 1). Because the activators used in the two equipment are slightly different, the reference ranges are different.

The types of tests that can be performed with TEG include Native and Kaolin TEG as the basic tests, Rapid TEG which enables quick response by inducing responses with the tissue factors, heparinase method that shows results by removing the effects of heparin, and Heparinase method that shows results by removing the effects of heparin, and

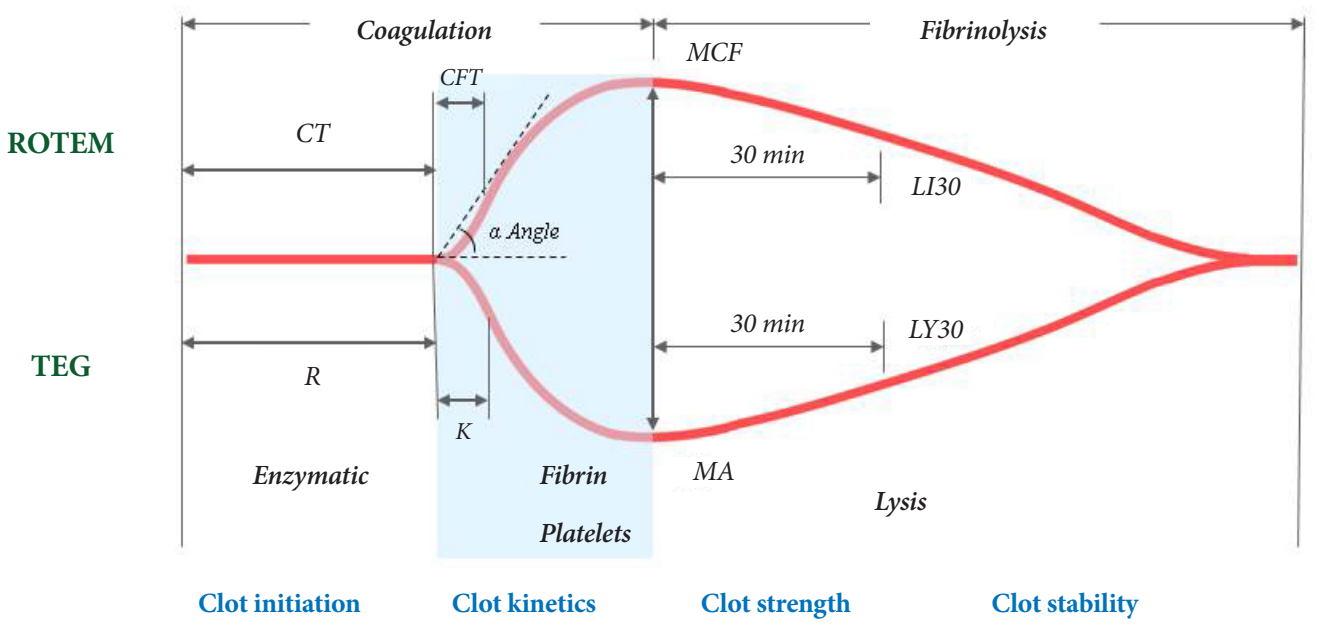

Fig. 1. Viscoelastic coagulation test using whole blood. CT: clotting time, R: reaction time, CFT: clot formation time, MCF: maximum clot firmness, MA: maximum amplitude. 
Functional Fibrinogen (FF) which shows the fibrin functions. In addition, the platelet function test is added. The tests that can be performed with ROTEM include NATEM which is a basic test that adds calcium to the citrated blood. The remaining tests can be classified into two types: EXTEM group and INTEM group. EXTEM is a method of making the response speed faster by activating the coagulation process using the tissue factors. Through this method, the extrinsic pathway of the coagulation can be identified. By adding aprotinin to this test and inhibiting fibrinolysis, APTEM can be obtained. By adding cytochalasin D and inhibiting platelet functions, FIBTEM can be performed. The other family of tests is INTEM by which the intrinsic pathway of the blood coagulation can be identified by adding ellagic acid. HEPTEM can be performed by adding heparinase and neutralizing the effect of heparin (Table 1). A test that can separately assess the platelet coagulation is soon to be commercialized. Both TEG and ROTEM were previously used by putting the blood in the testing cup and attaching it to the machine, the convenience of testing has increased presently with the introduction of the cartridge-type models.

Although the reference ranges of clotting time varies between testing equipment and method, plasma coagulation factor deficiency can generally be assessed within $5 \mathrm{~min}$. Abnormal clot strength can be assessed within $25 \mathrm{~min}$ if the values at 5,10 , or $20 \mathrm{~min}$ after clotting time are used before the maximum amplitude is observed. Additionally, by interpreting the amplitude results of FIBTEM or FF together, we could discriminate between the fibrin and platelet function or quantity problems. Although it can take more than one hour to completely assess the state of fibrinolysis, prediction can be made within $35 \mathrm{~min}$ if the abnormal fibrinolysis is severe by referencing the values at 30 min after the clotting time. In other words, using the viscoelastic coagulation test enables the assessment of the urgent need for fresh frozen plasma within $5 \mathrm{~min}$, the need for fibrin or platelet supplement other than the coagulation factors and the risk of bleeding within $25 \mathrm{~min}$.

\section{NECESSITY AND ADVANTAGES OF VISCOELASTIC COAGULATION TEST FOR LIVER TRANSPLANTATION}

Because patients undergoing liver transplantation have severely reduced liver functions, they are unable to generate sufficient amounts of vitamin K-dependent procoagulants, protein $\mathrm{C}$, and protein $\mathrm{S}$, which play important roles

Table 1. Description of ROTEM \& TEG Assays

\begin{tabular}{|c|c|c|}
\hline Test & & Description \\
\hline \multicolumn{3}{|l|}{ ROTEM } \\
\hline NATEM & & $\begin{array}{l}\text { Native whole blood sample analyzed following only recalcification } \\
\text { Impractical for clinical use given long CFT time }\end{array}$ \\
\hline \multirow[t]{4}{*}{ EXTEM } & & $\begin{array}{l}\text { Tissue factor activation: reagent contains tissue factor as an activator and provides information similar to } \\
\text { that of the PT }\end{array}$ \\
\hline & APTEM & $\begin{array}{l}\text { Contains aprotinin for inhibiting fibrinolysis; used in conjunction with EXTEM reagent and compared to } \\
\text { EXTEM analysis to assess fibrinolysis }\end{array}$ \\
\hline & FIBTEM & Utilizes cytochalasin $\mathrm{D}$, an actin polymerization inhibitor to exclude the platelet contribution \\
\hline & & $\begin{array}{l}\text { When compared to EXTEM analysis, allows qualitative analysis of the fibrinogen contribution to clot } \\
\text { strength }\end{array}$ \\
\hline \multirow[t]{2}{*}{ INTEM } & & $\begin{array}{l}\text { Contact activation: Reagent contains phospholipid and ellagic acid as activators and provides informa- } \\
\text { tion similar to that of the aPTT }\end{array}$ \\
\hline & HEPTEM & $\begin{array}{l}\text { Contains lyophilized heparinase for neutralizing unfractionated heparin; used in conjunction with INTEM } \\
\text { reagent and compared to ITEM analysis to assess heparin effect }\end{array}$ \\
\hline \multicolumn{3}{|l|}{ TEG } \\
\hline Kaolin & & $\begin{array}{l}\text { An intrinsic pathway activated assay identifies underlying hemostatic characteristics and risk of bleeding } \\
\text { or thrombosis }\end{array}$ \\
\hline Kaolin with heparinase & & $\begin{array}{l}\text { Eliminates the effect of heparin in the test sample, and used in conjunction with Kaolin assess the pres- } \\
\text { ence of systemic heparin }\end{array}$ \\
\hline Rapid TEG ${ }^{\text {TM }}$ & & $\begin{array}{l}\text { An intrinsic and extrinsic pathway activated assay speeds the coagulation process to more rapidly assess } \\
\text { coagulation properties }\end{array}$ \\
\hline Functional fibrinogen & & $\begin{array}{l}\text { Used in conjunction with Kaolin or Rapid TEG assess relative contribution of platelets and fibrin to overall } \\
\text { clot strength. }\end{array}$ \\
\hline
\end{tabular}

CFT: clot formation time, PT: prothrombin time, aPTT: activated partial prothrombin time. 
in coagulation. Furthermore, they simultaneously show decreased alpha-2-antiplasmin, thrombin activatable fibrinolysis inhibitor (TAFI), and plasminogen and increased tissue plasminogen activator (tPA) and plasminogen activator inhibitor-1 (PAI-1) which must be removed by the liver. Besides deficiency in liver functions, decrease in platelet count and its functions can be observed due to spleen enlargement and bone marrow suppression in patients with liver diseases. In addition, decrease in fibrinogen and its functions are common. These changes in the coagulation-related substances maintain an equilibrium but are unstable. Therefore, coagulopathy disorders and the resulting worsening of bleeding during liver transplantation is common. Because pre-operative CCT only reflects the pro-coagulant aspects of the plasma, it cannot provide accurate information, and the results are not related to the volume of bleeding or the transfusion of blood products during liver transplantation. Thus, it is difficult to predict bleeding or the need for blood transfusion $[9,10]$. Furthermore, because there are severe changes in the state of coagulation among the stages of surgery during liver transplantation, perioperative real-time monitoring is more important than the results from pre-operative tests. CCT requires the process of obtaining blood and sending it to a diagnostic lab, and generally 45-90 $\mathrm{min}$ are required to obtain the results. Therefore, CCT is not suitable for point-ofcare testing during liver transplantation. In contrast, the viscoelastic coagulation test enables point-of-care testing. It can lessen the burden of sending the blood to the lab, as long as the testing equipment is available, and generally 15-25 min are sufficient to obtain the main results for treatment. Therefore, it enables real-time monitoring and goal-directed therapy [11]. Although it can take 60-90 min to obtain all results, only few minutes are necessary to predict an immediate coagulopathy and clinically assess the need for treatment and the type of treatment. Many studies suggest that the viscoelastic coagulation test is more sensitive to coagulation disorders than CCT $[12,13]$. Moreover, in vivo studies on liver transplantation showed that pre-operative ROTEM test results are good predictors of the need for blood transfusion, particularly the need for fresh frozen plasma blood transfusion [14]. A retrospective analysis of patients undergoing liver transplantation reported that the MCF was strongly correlated with platelet count and fibrin concentration, and thus, can replace conventional tests [15]. In particular, because the amplitude obtained 5 or 10 min after the clotting time reflect the decrease in platelet or fibrin concentration, a quick assessment of the clot strength is possible [16]. Additionally, the viscoelastic test showed better results than CCT in predicting bleeding after the liver transplantation due to pathologic coagulopathy [17]. Thus, viscoelastic test is often used to test the coagulation function of the patients undergoing liver transplantation. It was reported in the 1980s by the University of Pittsburgh that blood transfusion can be decreased by applying TEG during liver transplantation [18]. A decrease in the transfusion of fresh frozen plasma by applying the transfusion strategy using this viscoelastic coagulation test for liver transplantation was also reported recently [19]. Because this test enables the immediate assessment of the state of fibrinolysis which cannot be obtained through CCT, it is suggested that transfusion may be decreased if the viscoelastic coagulation test is applied for liver transplantation where hyperfibrinolysis commonly occurs [20].

Depending on the type of the liver diseases that caused the hepatic failure, the state of coagulopathy may differ among patients undergoing liver transplantation. For example, patients with hepatocellular carcinoma, cholestatic hepatitis, and non-alcoholic steatohepatitis (NASH) show a relative hypercoagulation. In these patients, thrombosis is as important as bleeding. Although the prevalence of thrombosis is not high in Korea, liver disease and liver transplantation are clear risk factors for thromboembolism. Even if there is a bleeding tendency due to a coagulopathy, the risk of thrombosis does not decrease [21]. Furthermore, patients undergoing organ transplantation have a risk of fibrinolytic shutdown [22], and this is reported to increase the risk of thrombosis and bleeding during surgery. Krzanicki et al. [23] showed that many patients suffer from hypercoagulation during liver transplantation. Data on the resulting pulmonary embolism in liver transplantation showed that although the incidence was not high, the mortality rate was high, and it was particularly common just before or after graft reperfusion [24]. Therefore, diagnosing hypercoagulation state during liver transplantation is an important issue. According to the prospective study by McCrath et al. [25], the increase in MA is an independent predictive factor for post-operative acute myocardial infarction. Another research using ROTEM by Hincker et al. [26] showed that the increase in MCF can predict thrombosis. This emphasizes the need for viscoelastic coagulation test in liver transplantation.

However, some studies reported that there were no differences in bleeding or blood transfusion before and after 
using this coagulation testing [27]. It can be difficult to definitely conclude whether applying the viscoelastic coagulation test to the blood transfusion strategy during liver transplantation can really improve patient prognosis. However, this test has the clear advantage of reflecting the overall process of coagulation, from clot formation to fibrinolysis, and it can more sensitively and accurately diagnose coagulopathy than CCT during the surgery. For this reason, the application of viscoelastic coagulation test for liver transplantation can reduce bleeding and blood transfusion. Therefore, the Korean guideline for perioperative blood transfusion [28] and the guideline by the American Society of Anesthesiologists [29] consider the blood transfusion strategies using TEG and ROTEM to be important for not only liver transplantation but also perioperative blood management. Moreover, the guidelines for management of severe perioperative bleeding published by the European Society of Anaesthesiology [30] state that CCT is not suitable for surgeries in patients with liver disease and recommend TEG or ROTEM to diagnose perioperative coagulation disorders. Although further study is necessary, this recommendation based on that, viscoelastic coagulation test can improve prognosis by reducing blood transfusion, facilitate targeted management and can identify hyperfibrinolysis.

\section{BLOOD TRANSFUSION STRATEGY USING VISCOELASTIC COAGULATION TEST FOR LIVER TRANSPLANTATION}

To date, there is no protocol that applies viscoelastic coagulation test, which was found to be clearly superior as blood transfusion strategies for liver transplantation. A protocol published in 1993 [18] recommended the transfusion of 2-4 units of fresh frozen plasma if $\mathrm{R}$ time, which reflects the start of coagulation, is delayed by 15 minutes or more; transfusion of $1 \mathrm{unit} / 10 \mathrm{~kg}$ of platelet if MA, which reflects the clot strength, is thinner than $40 \mathrm{~mm}$; and transfusion of 6-12 units of cryoprecipitate if these blood transfusions do not improve blood coagulation or if the $a$ angle is less than 40 degrees. Findings from research in 2012 showed that using higher TEG threshold for blood transfusion than suggested in this protocol did not increase bleeding [31]. Because there are large variances in the indicators of the coagulation test during liver transplantation, the protocols of the tests are significantly related but not highly consistent [32]. Moreover, since protocols applied in the studies vary, it is difficult to conclude which method was the best. Additionally, the reference values of viscoelastic coagulation test are just average values of healthy subjects. Thus, values that are outside of this range does not necessarily suggest a coagulation disorder. Because values outside the reference range is not directly linked to bleeding, it is difficult to make a protocol based on the test values. In particular, in situations such as liver transplantations in which the state of coagulation changes rapidly, it is not appropriate to perform treatment based only on test results, and it is essential to consider the clinical situation. Research on patients undergoing liver transplantation showed that the predicted cut-off values of risk for bleeding or blood transfusion were within the reference range which further emphasized that treatment decisions based only on test results are inappropriate [14].

Severe fluctuations in the coagulation status appear at each phase of liver transplantation surgery (Fig. 2). Deficiency or functional decline in blood coagulation factor and platelet is typical for the preanhepatic phase. Therefore, thin graphs with extended R and CT and low values of MA and MCF are common. Because it better reflects the balanced coagulation state, stable results can be seen on the viscoelastic test even in patients with severe coagulopathy according to the CCT in the beginning of the surgery. On the other hand, with the progression of the surgery, the worsening of the coagulopathy can be observed in real-time by viscoelastic test. Therefore, while unnecessary plasma transfusion is possible when using only the palliative coagulation test results in the beginning of the surgery, this can be prevented by applying the viscoelastic coagulation test and a treatment can be performed when it is really needed. In the anhepatic phase, the previously mentioned coagulopathy worsens and tPA is not eliminated, and thus, hyperfibrinolysis can occur. Thus, LI30, LI60, LY30, and LY60 increase or worsen. Here, an appropriate administration of an anti-fibrinolysis medication (tranexamic acid) can prevent the worsening of bleeding. In particular, all previously discussed coagulation disorders worsen at the end of the anhepatic phase and immediately after reperfusion, due to the increase in TPA and PAI-1, decrease in alpha-2-antiplasmin, and the influx of heparin-like substances in the donated liver [33-35]. Although coagulopathy is observed in combination, referencing the changes in each value and the results of fibrinogen and platelet functions from the viscoelastic test can be of much help in assessing the most urgently needed treatment. In the neohe- 


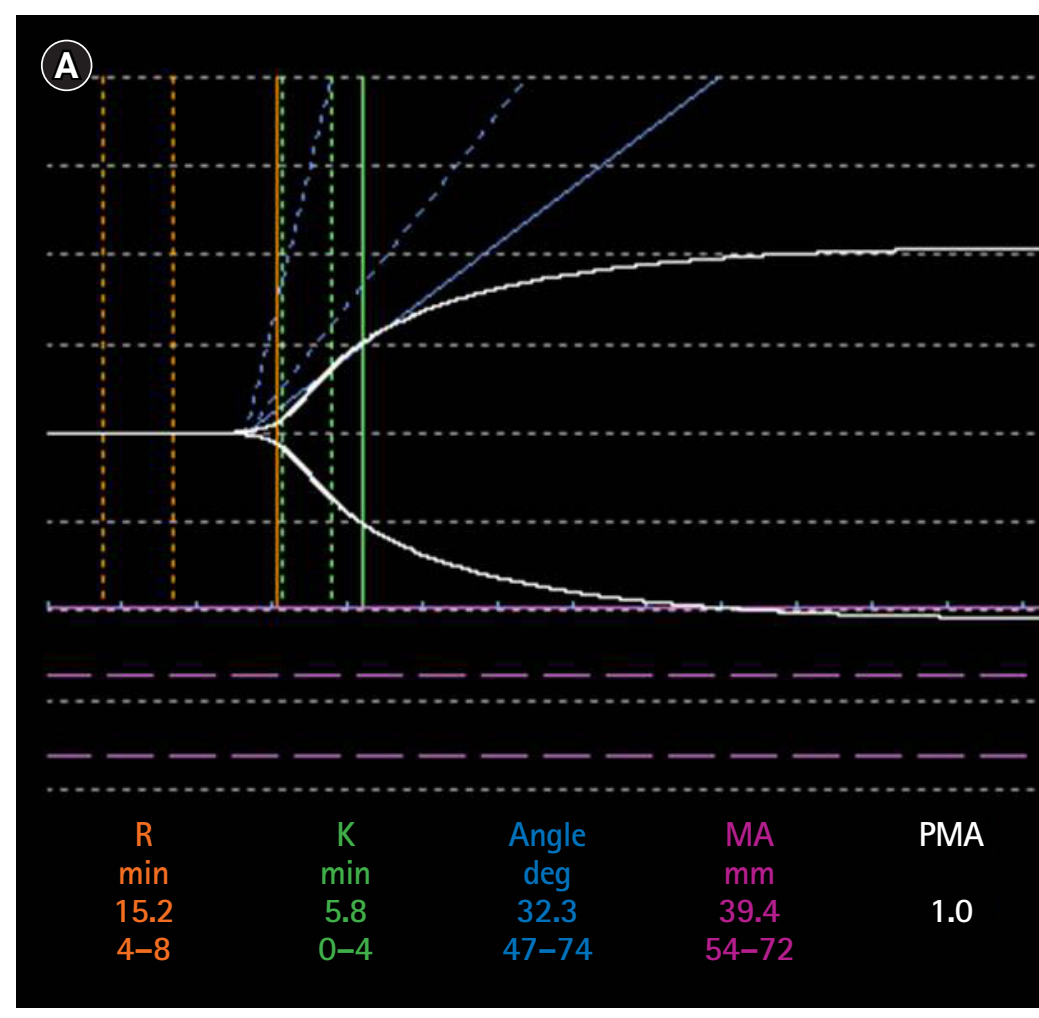

Sample data:

Normal range:

\begin{tabular}{|c|c|c|c|}
\hline$R$ & 15.2 & $\min$ & $4-8$ \\
\hline $\mathrm{K}$ & 5.8 & $\mathrm{~min}$ & $0-4$ \\
\hline Angle & 32.3 & $\mathrm{deg}$ & $47-74$ \\
\hline $\mathrm{MA}$ & 39.4 & $\mathrm{~mm}$ & $54-72$ \\
\hline PMA & 1.0 & & \\
\hline $\mathrm{G}$ & $3.2 \mathrm{~K}$ & $\mathrm{~d} / \mathrm{sc}$ & $6.0 \mathrm{~K}-13.2 \mathrm{~K}$ \\
\hline $\mathrm{EPL}$ & 0.0 & $\%$ & $0-15$ \\
\hline $\mathrm{A}$ & 41.6 & $\mathrm{~mm}$ & \\
\hline $\mathrm{Cl}$ & -12.6 & & $-3-3$ \\
\hline $\mathrm{LY} 30$ & 0.0 & $\%$ & $0-8$ \\
\hline A30 & 39.4 & $\mathrm{~mm}$ & \\
\hline $\mathrm{CL30}$ & 100.0 & $\%$ & $92-100$ \\
\hline A60 & 38.7 & $\mathrm{~mm}$ & \\
\hline CL60 & 100.0 & $\%$ & $85-100$ \\
\hline LY60 & 0.0 & $\%$ & $0-15$ \\
\hline CLT & 33.7 & $\mathrm{~min}$ & \\
\hline TPI & 5.6 & $/ \mathrm{sec}$ & $32-527$ \\
\hline TMA & 42.4 & $\mathrm{~min}$ & \\
\hline $\mathrm{E}$ & 65.0 & $\mathrm{~d} / \mathrm{sc}$ & $120-264$ \\
\hline SP & 13.1 & $\mathrm{~min}$ & \\
\hline LTE & $>3 \mathrm{~h}$ & $\mathrm{~min}$ & \\
\hline & & & \\
\hline
\end{tabular}

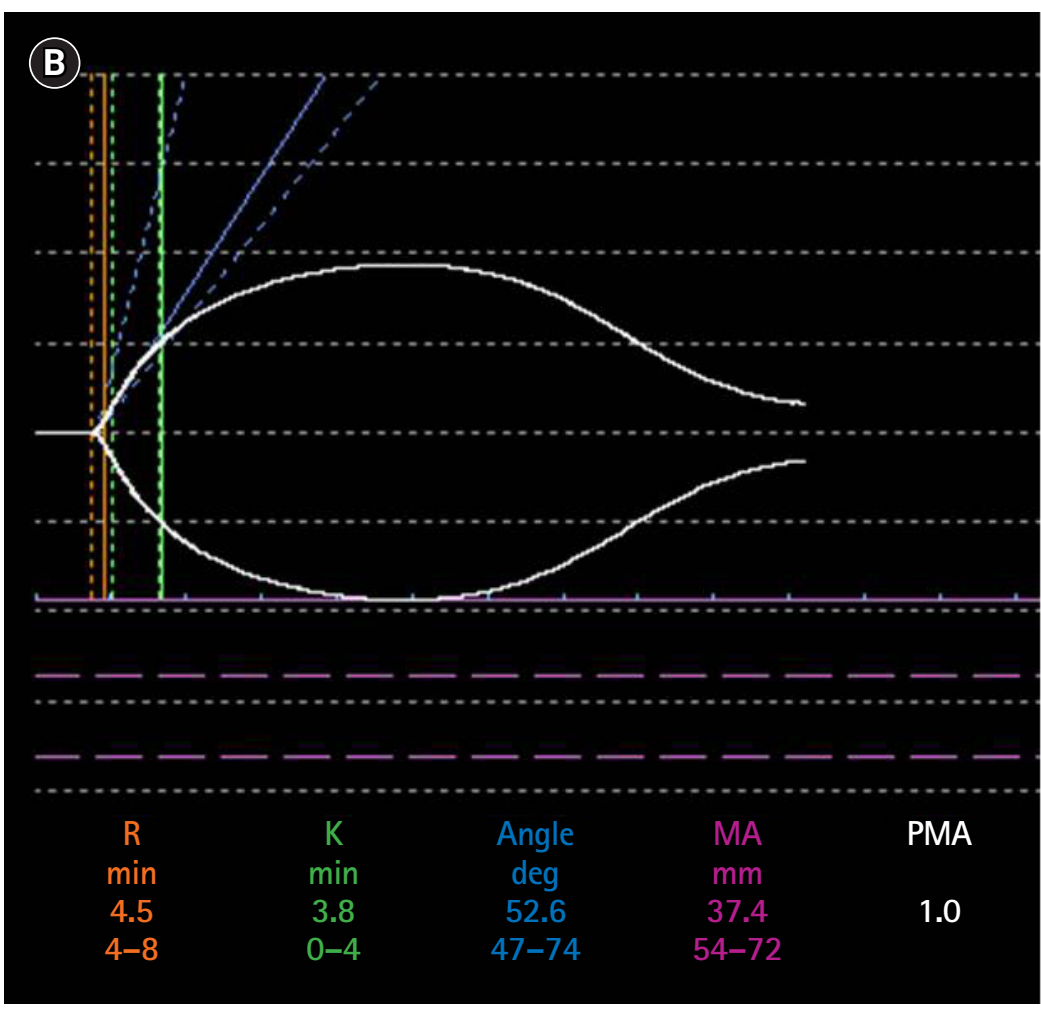

Sample data:

Normal range:

\begin{tabular}{|c|c|c|c|}
\hline $\mathrm{R}$ & 4.5 & $\mathrm{~min}$ & $4-8$ \\
\hline $\mathrm{K}$ & 3.8 & $\mathrm{~min}$ & $0-4$ \\
\hline Angle & 52.6 & $\mathrm{deg}$ & $47-74$ \\
\hline $\mathrm{MA}$ & 37.4 & $\mathrm{~mm}$ & $54-72$ \\
\hline PMA & 1.0 & & \\
\hline $\mathrm{G}$ & $3.0 \mathrm{~K}$ & $\mathrm{~d} / \mathrm{sc}$ & $6.0 \mathrm{~K}-13.2 \mathrm{~K}$ \\
\hline EPL & 34.1 & $\%$ & $0-15$ \\
\hline $\mathrm{A}$ & 6.3 & $\mathrm{~mm}$ & \\
\hline $\mathrm{Cl}$ & -3.6 & & $-3-3$ \\
\hline LY30 & 34.1 & $\%$ & $0-8$ \\
\hline A30 & 6.6 & $\mathrm{~mm}$ & \\
\hline CL30 & 17.6 & $\%$ & $92-100$ \\
\hline A60 & 6.3 & $\mathrm{~mm}$ & \\
\hline CL60 & 16.8 & $\%$ & $85-100$ \\
\hline LY60 & 34.2 & $\%$ & $0-15$ \\
\hline CLT & 31.1 & $\mathrm{~min}$ & \\
\hline TPI & 7.8 & $/ \mathrm{sec}$ & $32-527$ \\
\hline TMA & 20.2 & $\mathrm{~min}$ & \\
\hline $\mathrm{E}$ & 59.7 & $\mathrm{~d} / \mathrm{sc}$ & $120-264$ \\
\hline SP & 4.0 & $\mathrm{~min}$ & \\
\hline LTE & 33.6 & $\mathrm{~min}$ & \\
\hline & & & \\
\hline
\end{tabular}

Fig. 2. Examples of thromboelastography during liver transplantation. (A) Preanhepatic phase. (B) Anhepatic phase. (C) Five minutes after reperfusion. (D) Neohepatic phase. R: reaction time, MA: maximum amplitude (Continued to the next page). 


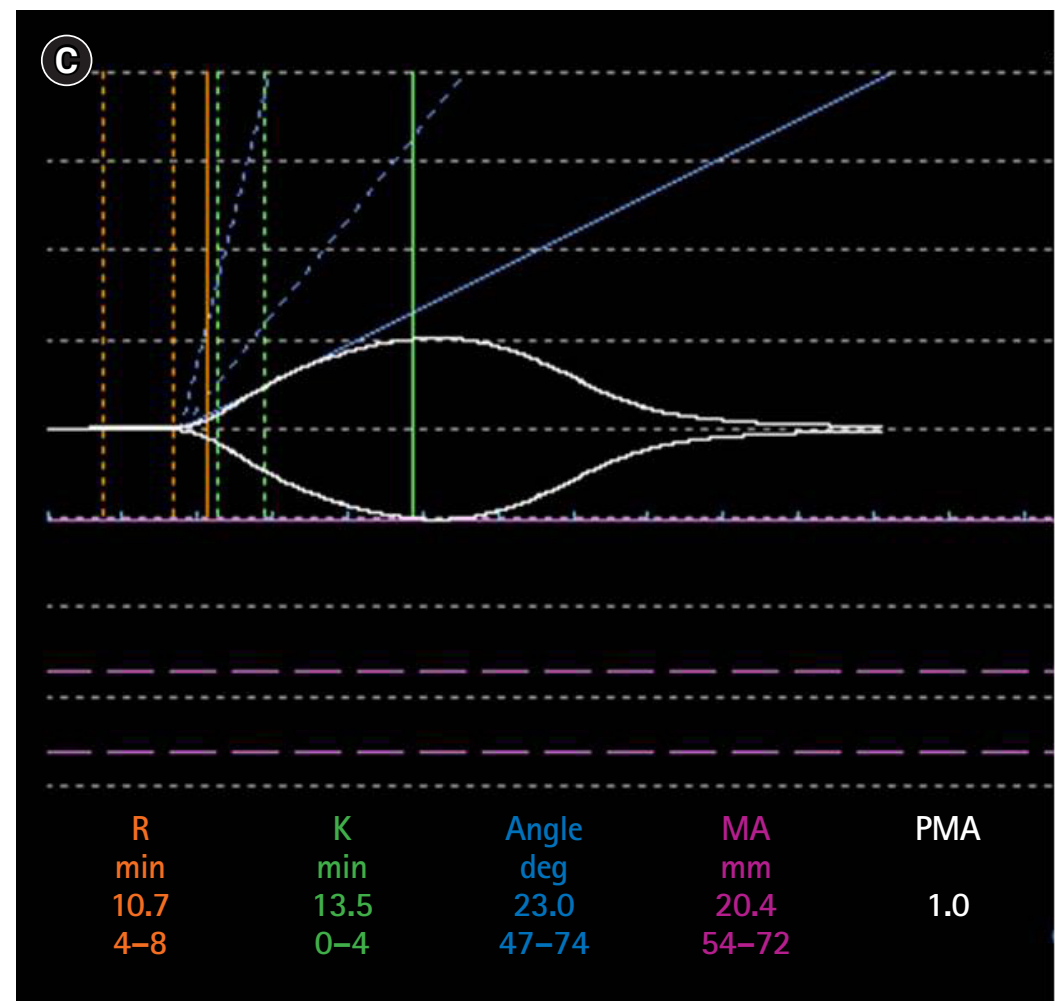

Sample data:

Normal range:

\begin{tabular}{|c|c|c|c|}
\hline$R$ & 10.7 & $\min$ & $4-8$ \\
\hline $\mathrm{K}$ & 13.5 & $\mathrm{~min}$ & $0-4$ \\
\hline Angle & 23.0 & $\mathrm{deg}$ & $47-74$ \\
\hline $\mathrm{MA}$ & 20.4 & $\mathrm{~mm}$ & $54-72$ \\
\hline PMA & 1.0 & & \\
\hline $\mathrm{G}$ & $1.3 \mathrm{~K}$ & $\mathrm{~d} / \mathrm{sc}$ & $6.0 \mathrm{~K}-13.2 \mathrm{~K}$ \\
\hline $\mathrm{EPL}$ & 65.7 & $\%$ & $0-15$ \\
\hline $\mathrm{A}$ & 0.6 & $\mathrm{~mm}$ & \\
\hline $\mathrm{Cl}$ & -15.6 & & $-3-3$ \\
\hline LY30 & 65.7 & $\%$ & $0-8$ \\
\hline A30 & 0.6 & $\mathrm{~mm}$ & \\
\hline CL30 & 2.9 & $\%$ & $92-100$ \\
\hline A60 & 0.6 & $\mathrm{~mm}$ & \\
\hline CL60 & 2.9 & $\%$ & $85-100$ \\
\hline LY60 & 62.3 & $\%$ & $0-15$ \\
\hline CLT & 20.2 & $\mathrm{~min}$ & \\
\hline TPI & 0.9 & $/ \mathrm{sec}$ & $32-527$ \\
\hline TMA & 24.7 & $\mathrm{~min}$ & \\
\hline $\mathrm{E}$ & 25.6 & $\mathrm{~d} / \mathrm{sc}$ & $120-264$ \\
\hline SP & 8.9 & $\mathrm{~min}$ & \\
\hline LTE & 20.2 & $\mathrm{~min}$ & \\
\hline & & & \\
\hline
\end{tabular}

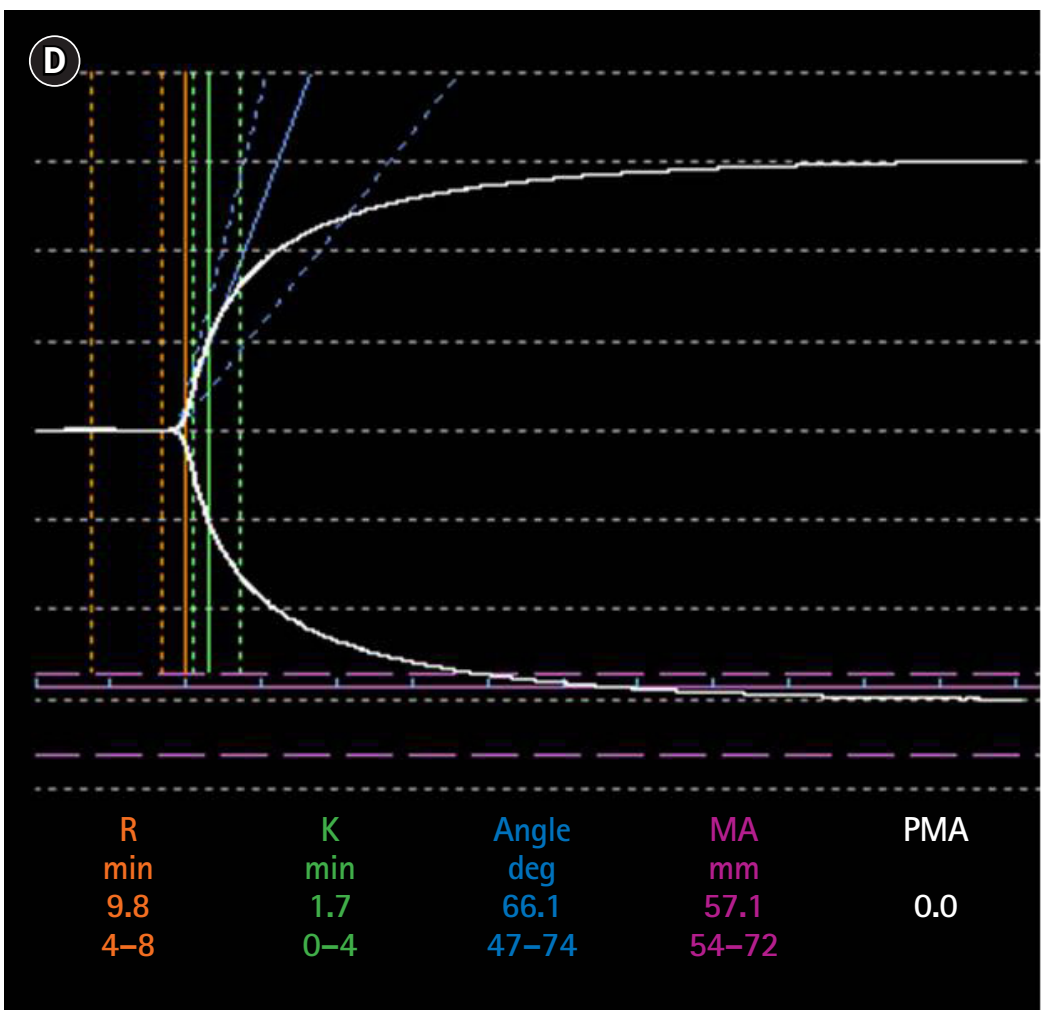

Sample data:

Normal range:

\begin{tabular}{|c|c|c|c|}
\hline$R$ & 9.8 & $\min$ & $4-8$ \\
\hline $\mathrm{K}$ & 1.7 & $\mathrm{~min}$ & $0-4$ \\
\hline Angle & 66.1 & $\mathrm{deg}$ & $47-74$ \\
\hline $\mathrm{MA}$ & 57.1 & $\mathrm{~mm}$ & $54-72$ \\
\hline PMA & 0.0 & & \\
\hline $\mathrm{G}$ & $6.6 \mathrm{~K}$ & $\mathrm{~d} / \mathrm{sc}$ & $6.0 \mathrm{~K}-13.2 \mathrm{~K}$ \\
\hline $\mathrm{EPL}$ & 0.0 & $\%$ & $0-15$ \\
\hline $\mathrm{A}$ & 60.1 & $\mathrm{~mm}$ & \\
\hline $\mathrm{Cl}$ & -2.8 & & $-3-3$ \\
\hline LY30 & 0.0 & $\%$ & $0-8$ \\
\hline A30 & 57.1 & $\mathrm{~mm}$ & \\
\hline CL30 & 100.0 & $\%$ & $92-100$ \\
\hline A60 & 57.1 & $\mathrm{~mm}$ & \\
\hline CL60 & 100.0 & $\%$ & $85-100$ \\
\hline LY60 & 0.0 & $\%$ & $0-15$ \\
\hline CLT & 30.8 & $\mathrm{~min}$ & \\
\hline TPI & 39.9 & $/ \mathrm{sec}$ & $32-527$ \\
\hline TMA & 34.4 & $\mathrm{~min}$ & \\
\hline $\mathrm{E}$ & 133.0 & $\mathrm{~d} / \mathrm{sc}$ & $120-264$ \\
\hline SP & 9.3 & $\mathrm{~min}$ & \\
\hline LTE & $>3 \mathrm{~h}$ & $\mathrm{~min}$ & \\
\hline & & & \\
\hline
\end{tabular}

Fig. 2. (Continued from the previous page). 
patic phase, the coagulopathy can improve due to the functions of the transplanted liver, and this is reflected in the viscoelastic coagulation test results. If the coagulopathy appears unchanged or worsened according to the viscoelastic coagulation test in this phase, the possibility of excessive bleeding must be considered even if this is a generally stable period; in addition, the possibility that the transplanted liver is dysfunctional must be considered as well.

\section{CONCLUSION}

Viscoelastic coagulation test is a point-of-care testing that can show not only the beginning of the coagulation but also the clot strength and stability. It enables faster judgment and treatment than CCT. As its utility has already been shown in clinical situations with coagulation disorders and severe bleeding such as trauma using both clinical and academic studies, this method can be used effectively for liver transplantation which has a high risk of bleeding. Through further research, it is anticipated that this test can be used as one of the standardized patient blood management strategies for liver transplantation.

\section{SUPPLEMENTARY MATERIALS}

Supplementary data containing Korean version of this article is available at https://doi.org/10.17085/apm.2020. 15.2.143.

\section{CONFLICTS OF INTEREST}

No potential conflict of interest relevant to this article was reported.

\section{ORCID}

Sun Young Park, https://orcid.org/0000-0003-2588-3324

\section{REFERENCES}

1. Miki C, Iriyama K, Gunson BK, Mayer AD, Buckels JA, McMaster P. Influence of intraoperative blood loss on plasma levels of cytokines and endotoxin and subsequent graft liver function. Arch Surg 1997; 132: 136-41.

2. Abdel-Wahab OI, Healy B, Dzik WH. Effect of fresh-frozen plasma transfusion on prothrombin time and bleeding in patients with mild coagulation abnormalities. Transfusion 2006; 46 :
1279-85.

3. Chowdary P, Saayman AG, Paulus U, Findlay GP, Collins PW. Efficacy of standard dose and $30 \mathrm{ml} / \mathrm{kg}$ fresh frozen plasma in correcting laboratory parameters of haemostasis in critically ill patients. Br J Haematol 2004; 125: 69-73.

4. Plotkin AJ, Wade CE, Jenkins DH, Smith KA, Noe JC, Park MS, et al. A reduction in clot formation rate and strength assessed by thrombelastography is indicative of transfusion requirements in patients with penetrating injuries. J Trauma 2008; 64(2 Suppl): S64-8.

5. Fries D, Innerhofer P, Schobersberger W. Time for changing coagulation management in trauma-related massive bleeding. Curr Opin Anaesthesiol 2009; 22: 267-74.

6. Johansson PI, Stissing T, Bochsen L, Ostrowski SR. Thrombelastography and tromboelastometry in assessing coagulopathy in trauma. Scand J Trauma Resusc Emerg Med 2009; 17: 45.

7. Kim GS. Thromboelastography. Korean J Anesthesiol 2004; 47: 297-304.

8. Mallett SV. Clinical utility of viscoelastic tests of coagulation (TEG/ROTEM) in patients with liver disease and during liver transplantation. Semin Thromb Hemost 2015; 41: 527-37.

9. Findlay JY, Rettke SR. Poor prediction of blood transfusion requirements in adult liver transplantations from preoperative variables. J Clin Anesth 2000; 12: 319-23.

10. Massicotte L, Beaulieu D, Roy JD, Marleau D, Vandenbroucke F, Dagenais $\mathrm{M}$, et al. MELD score and blood product requirements during liver transplantation: no link. Transplantation 2009; 87: 1689-94.

11. Watson GA, Sperry JL, Rosengart MR, Minei JP, Harbrecht BG, Moore EE, et al. Inflammation and Host Response to Injury Investigators. Fresh frozen plasma is independently associated with a higher risk of multiple organ failure and acute respiratory distress syndrome. J Trauma 2009; 67: 221-7.

12. Davenport R, Manson J, De'Ath H, Platton S, Coates A, Allard S, et al. Functional definition and characterization of acute traumatic coagulopathy. Crit Care Med 2011; 39: 2652-8.

13. Woolley T, Midwinter M, Spencer P, Watts S, Doran C, Kirkman E. Utility of interim ROTEM $^{\circ}$ values of clot strength, A5 and A10, in predicting final assessment of coagulation status in severely injured battle patients. Injury 2013; 44: 593-9.

14. Fayed N, Mourad W, Yassen K, Görlinger K. Preoperative thromboelastometry as a predictor of transfusion requirements during adult living donor liver transplantation. Transfus Med Hemother 2015; 42: 99-108.

15. Jeong SM, Song JG, Seo H, Choi JH, Jang DM, Hwang GS. Quantification of both platelet count and fibrinogen concentration using maximal clot firmness of thromboelastometry during liver transplantation. Transplant Proc 2015; 47: 1890-5.

16. Song JG, Jeong SM, Jun IG, Lee HM, Hwang GS. Five-minute 
parameter of thromboelastometry is sufficient to detect thrombocytopenia and hypofibrinogenaemia in patients undergoing liver transplantation. Br J Anaesth 2014; 112: 290-7.

17. Dötsch TM, Dirkmann D, Bezinover D, Hartmann M, Treckmann JW, Paul A, et al. Assessment of standard laboratory tests and rotational thromboelastometry for the prediction of postoperative bleeding in liver transplantation. Br J Anaesth 2017; 119: 402-10.

18. Kang YG, Martin DJ, Marquez J, Lewis JH, Bontempo FA, Shaw BW Jr, et al. Intraoperative changes in blood coagulation and thrombelastographic monitoring in liver transplantation. Anesth Analg 1985; 64: 888-96.

19. Wang SC, Shieh JF, Chang KY, Chu YC, Liu CS, Loong CC, et al. Thromboelastography-guided transfusion decreases intraoperative blood transfusion during orthotopic liver transplantation: randomized clinical trial. Transplant Proc 2010; 42: 2590-3.

20. Trzebicki J, Flakiewicz E, Kosieradzki M, Blaszczyk B, Kołacz M, Jureczko L, et al. The use of thromboelastometry in the assessment of hemostasis during orthotopic liver transplantation reduces the demand for blood products. Ann Transplant 2010; 15: 19-24.

21. Dabbagh O, Oza A, Prakash S, Sunna R, Saettele TM. Coagulopathy does not protect against venous thromboembolism in hospitalized patients with chronic liver disease. Chest 2010; 137: 1145-9.

22. Raveh Y, Souki F, Livingstone J, Beduschi T, Vianna R, Rodriguez Y, et al. Fibrinolytic shutdown is associated with intraoperative thrombosis and hemorrhage during visceral transplant. Semin Cardiothorac Vasc Anesth 2019; 23: 300-8.

23. Krzanicki D, Sugavanam A, Mallett S. Intraoperative hypercoagulability during liver transplantation as demonstrated by thromboelastography. Liver Transpl 2013; 19: 852-61.

24. Warnaar N, Molenaar IQ, Colquhoun SD, Slooff MJ, Sherwani S, de Wolf AM, et al. Intraoperative pulmonary embolism and intracardiac thrombosis complicating liver transplantation: a systematic review. J Thromb Haemost 2008; 6: 297-302.

25. McCrath DJ, Cerboni E, Frumento RJ, Hirsh AL, Bennett-Guerrero E. Thromboelastography maximum amplitude predicts postoperative thrombotic complications including myocardial infarction. Anesth Analg 2005; 100: 1576-83.
26. Hincker A, Feit J, Sladen RN, Wagener G. Rotational thromboelastometry predicts thromboembolic complications after major non-cardiac surgery. Crit Care 2014; 18: 549.

27. Roullet S, Freyburger G, Cruc M, Quinart A, Stecken L, Audy M, et al. Management of bleeding and transfusion during liver transplantation before and after the introduction of a rotational thromboelastometry-based algorithm. Liver Transpl 2015; 21: $169-79$

28. Koo BN, Kwon MA, Kim SH, Kim JY, Moon YJ, Park SY, et al. Korean clinical practice guideline for perioperative red blood cell transfusion from Korean Society of Anesthesiologists. Korean J Anesthesiol 2019; 72: 91-118.

29. American Society of Anesthesiologists Task Force on Perioperative Blood Management. Practice guidelines for perioperative blood management: an updated report by the American Society of Anesthesiologists Task Force on Perioperative Blood Management. Anesthesiology 2015; 122: 241-75.

30. Kozek-Langenecker SA, Afshari A, Albaladejo P, Santullano CA, De Robertis E, Filipescu DC, et al. Management of severe perioperative bleeding: guidelines from the European Society of Anaesthesiology. Eur J Anaesthesiol 2013; 30: 270-382.

31. Wang SC, Lin HT, Chang KY, Mandell MS, Ting CK, Chu YC, et al. Use of higher thromboelastogram transfusion values is not associated with greater blood loss in liver transplant surgery. Liver Transpl 2012; 18: 1254-8.

32. Coakley M, Reddy K, Mackie I, Mallett S. Transfusion triggers in orthotopic liver transplantation: a comparison of the thromboelastometry analyzer, the thromboelastogram, and conventional coagulation tests. J Cardiothorac Vasc Anesth 2006; 20: 548-53.

33. Kettner SC, Gonano C, Seebach F, Sitzwohl C, Acimovic S, Stark J, et al. Endogenous heparin-like substances significantly impair coagulation in patients undergoing orthotopic liver transplantation. Anesth Analg 1998; 86: 691-5.

34. Kang Y. Coagulation and liver transplantation. Transplant Proc 1993; 25: 2001-5.

35. Bakker CM, Metselaar HJ, Groenland TN, Gomes MJ, Knot EA, Hesselink EJ, et al. Increased tissue-type plasminogen activator activity in orthotopic but not heterotopic liver transplantation: the role of the anhepatic period. Hepatology 1992; 16: 404-8. 